\title{
Analytical Solution for MHD Flow of a Magnetic Fluid within a Thick Porous Annulus
}

\author{
Shihhao Yeh, ${ }^{1}$ Tsai-Jung Chen, ${ }^{2}$ and Jik Chang Leong ${ }^{2}$ \\ ${ }^{1}$ Department of Engineering Science, National Cheng Kung University, Tainan City 70101, Taiwan \\ ${ }^{2}$ Department of Vehicle Engineering, National Pingtung University of Science and Technology, Neipu, Pingtung 91201, Taiwan
}

Correspondence should be addressed to Jik Chang Leong; jcleong@mail.npust.edu.tw

Received 27 June 2013; Accepted 2 April 2014; Published 11 June 2014

Academic Editor: Subhas Abel

Copyright (C) 2014 Shihhao Yeh et al. This is an open access article distributed under the Creative Commons Attribution License, which permits unrestricted use, distribution, and reproduction in any medium, provided the original work is properly cited.

\begin{abstract}
The steady-state problem of a magnetic fluid filling a porous annulus between two cylindrical walls under the influence of a nonuniform radially outward magnetic field has been investigated. The cylindrical walls are either electrically perfectly insulated or electrically perfectly conducting. The permeability of the porous annulus increases with its radial location. The governing partial differential equations were derived carefully and closed form solutions for the profiles of the velocity component and the induced magnetic component were obtained. The effect of the strength of the externally applied magnetic field, the permeability of the porous annulus, and the conductivity of the cylindrical walls were examined through the angular velocity components, as well as the induced magnetic field.
\end{abstract}

\section{Introduction}

The effects of magnetic field on an electrically conducting fluid have great importance because of their recent applications related to MHD generators [1,2], geothermal energy excitations [3, 4], plasma controls [5-7], MHD boundary layer controls [8-10], and so forth. The governing partial differential equations involved in MHD studies are well known for their nonlinearity; exact solutions are difficult to obtain unless the problem is very fundamental in conjunction with highly simplified conditions. Perhaps, MHD Couette flow is one of best examples. It deals with a conducting fluid filled between two infinite planes where an external magnetic field is applied across these planes. It has been confirmed that the presence of the external magnetic field induces a Lorentz force which either accelerates or decelerates the flow elements between the planes. Furthermore, the flow behaviors also depend strongly on the electrical properties of the planes [11]. The performances of the MHD flow in ducts and pipes have also been studied quite thoroughly [12-16].

On the other hand, Heiser and Shercliff [17] have developed the theory for the motion of a viscous conducting liquid in a pair of rotating long concentric cylinders subjected to a radial magnetic field. Later, Molokov and Allen [18] extended the aforementioned theory and more importantly they found a mean to decouple the equations. Tsai et al. [19] and Kuo and Leong [20] have extended the study for thin annulus. The effects of external magnetic or electric fields of various orientations have also been investigated extensively [21, 22].

Since 2005, Stokes' first problem in a porous space under the influence of an applied magnetic field has been attempted [23-25]. As an extension to Stokes' problems, Khan et al. $[26,27]$ solved the modified Darcy's law for a transient MHD flow in a similar setting. In additional to Stokes' problems in porous media, MHD Couette flow associated with porous media has also gained a lot of attention. Hayat et al. [28] employed HAM, a mathematical approach, to investigate the influence of a transverse magnetic field on the MHD Couette flow in a porous channel. Seth et al. [29] then considered the MHD flow within a porous channel whose motion was also induced by either an impulsive or a uniform acceleration of the channel bottom wall. Bég et al. [30] obtained a theoretical solution for the MHD Couette flow in a rotating horizontal parallel-plate channel filled with a highly permeable porous medium subjected to an inclined uniform magnetic field. At the same time, Ramana Murthy et al. [31] investigated the response of an unsteady MHD fluid through a circular pipe with porous walls in the presence of a transverse magnetic 


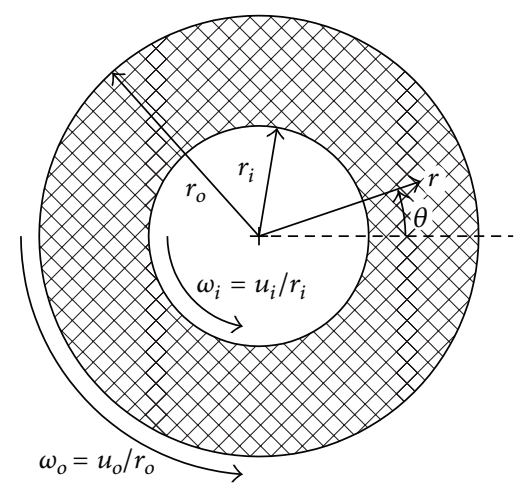

FIGURE 1: Schematics of present study.

field. Also, Khan et al. [32] theoretically obtained the velocity field for a fully developed MHD flow within a porous space in a circular pipe based on the modified Darcy's law. Srinivas and Muthuraj [33] studied the MHD flow in a vertical wavy porous medium in the presence of a temperature-dependent heat source.

This work aims to analyze the MHD flow of a generalized magnetic fluid in an annulus. The inner and outer cylinders enclosing the annular space are either electrically perfectly insulated or electrically perfectly conducting. The momentum of the rotating magnetic fluid originates from the shear effect by the rotating outer cylinder. In the beginning, the governing equations were carefully simplified and derived from the relevant Maxwell's equations as well as the Brinkman's extension of Darcy's law. The analytical solutions were then obtained for various boundary conditions to give the profiles of tangential velocity component and the induced magnetic field.

\section{Formulation}

The annular space that the present study considers is shown in Figure 1. The space considered here is confined between a pair of long concentric cylinders whose inner and outer radii are denoted by $r_{i}$ and $r_{o}$ but extends to infinity in the axial direction. The inner and outer cylinder surfaces are both adiabatic and therefore the problem is isothermal. At the same time, a radially outward magnetic field is applied externally over the annular space [34]. This externally applied magnetic field across the annulus is given as $\vec{B}=B_{0} / r \widehat{e}_{r}$. Therefore, the flow and magnetic fields within the annular are axis-symmetric. The conducting fluid considered is assumed incompressible, Newtonian, and so highly electrically conductive that no electric charge will accumulate within the fluid. In additional to the above conditions, it is also assumed that the flow is steady, one-dimensional, and laminar, while the gravitational force is negligible. The homogenous and isotropic porous annulus has no effect on the electric current, the magnetic field strength, and their distributions. Also, the wall effect and non-Darcian effects are ignored. Furthermore, the permeability of the porous annulus is assumed $K_{p}=$ Da $r^{2}$, where $\mathrm{Da}$ is the Darcy number whose definition will be discussed later. For a constant $\mathrm{Da}$, it is clear that $K_{p}$ varies with $r$. In other words, the porous annulus is more permeable far away from the center. The above assumptions simplifies the continuity and Navier-Stokes equations that appear to be

$$
\begin{gathered}
\nabla \cdot \vec{V}=0, \\
\rho \nu \nabla^{2} \vec{V}-\frac{\rho v}{K_{p}} \vec{V}+\vec{j} \times \vec{B}=0,
\end{gathered}
$$

where the last term in (2) is the Lorentz force. The magnetic field derived based on Maxwell's relations under a steadystate assumption can be simplified to yield the following magnetic field equation [35]:

$$
\nabla \times(\vec{V} \times \vec{B})+\frac{1}{\sigma \mu} \nabla^{2} \vec{B}=0
$$

With the introduction of the following dimensionless parameters

$$
\begin{gathered}
\bar{r}=\frac{r}{a}, \quad \bar{u}_{\theta}=\frac{u_{\theta}}{u_{o}}, \\
\bar{b}_{\theta}=\frac{b_{\theta}}{u_{o} \mu \sqrt{\sigma \nu \rho}}, \quad \mathrm{Ha}=B_{0} a \sqrt{\frac{\sigma}{\rho \nu} .}
\end{gathered}
$$

Equations (2) to (3) reduce to the following dimensionless magnetic induced equation and dimensionless magnetic momentum equation:

$$
\begin{gathered}
\mathrm{Ha} \frac{d}{d \bar{r}}\left(\frac{\bar{u}_{\theta}}{\bar{r}}\right)+\frac{d}{d \bar{r}}\left[\frac{1}{\bar{r}} \frac{d}{d \bar{r}}\left(\bar{r} \bar{b}_{\theta}\right)\right]=0, \\
\frac{\mathrm{Ha}}{\bar{r}^{2}} \frac{d}{d \bar{r}}\left(\bar{r} \bar{b}_{\theta}\right)+\frac{d^{2} \bar{u}_{\theta}}{d \bar{r}^{2}}+\frac{1}{\bar{r}} \frac{d \bar{u}_{\theta}}{d \bar{r}}-\left(\frac{1}{\mathrm{Da}}+1\right) \frac{\bar{u}_{\theta}}{\bar{r}^{2}}=0,
\end{gathered}
$$

where the bar indicates that the parameter beneath it is dimensionless, $a$ is the gap between the inner and outer cylinders (i.e., $a=r_{o}-r_{i}$ ), and $u_{0}$ is the linear velocity on the outer cylinder. Two of the additional dimensionless parameters that appeared in the above governing equations are the Darcy number $(\mathrm{Da})$ and the Hartmann number $(\mathrm{Ha})$. The former is an indication of the permeability of the porous media, while the latter signifies the relative importance between the magnetic and viscous forces.

The cylindrical walls considered in this work are assumed made of materials either perfectly insulated or perfectly conducting. The different combinations investigated include (a) both inner and outer cylinders are perfectly insulated and (b) only one of the cylinders is perfectly insulated, while the other is perfectly conducting. Also, the outer cylinder is assumed to rotate at a rotational speed which yields a linear velocity of $u_{o}$, whereas the inner cylinder rotates at an arbitrary rotational speed corresponding to a linear velocity of $u_{i}$. These conditions, for different cases, 
are mathematically described by the following dimensionless boundary conditions.

(a) Both the inner and outer cylinders are perfectly insulated:

$$
\begin{array}{lll}
\bar{r}=\frac{r_{i}}{a}=\bar{r}_{i}, & \bar{u}_{\theta}=\frac{u_{i}}{u_{o}}=\bar{u}_{i}, & \bar{b}_{\theta}=0, \\
\bar{r}=\frac{r_{o}}{a}=\bar{r}_{o}, & \bar{u}_{\theta}=\frac{u_{o}}{u_{o}}=1, & \bar{b}_{\theta}=0 .
\end{array}
$$

(b) The inner cylinder is perfectly insulated but the outer cylinder is perfectly conducting:

$$
\begin{aligned}
& \bar{r}=\frac{r_{i}}{a}=\bar{r}_{i}, \quad \bar{u}_{\theta}=\frac{u_{i}}{u_{0}}=\bar{u}_{i}, \quad \bar{b}_{\theta}=0, \\
& \bar{r}=\frac{r_{o}}{a}=\bar{r}_{o}, \quad \bar{u}_{\theta}=\frac{u_{o}}{u_{o}}=1, \quad \frac{\partial \bar{b}_{\theta}}{\partial \bar{r}}=0 .
\end{aligned}
$$

(c) The outer cylinder is perfectly insulated but the inner cylinder is perfectly conducting:

$$
\begin{array}{ccc}
\bar{r}=\frac{r_{i}}{a}=\bar{r}_{i}, & \bar{u}_{\theta}=\frac{u_{i}}{u_{o}}=\bar{u}_{i}, & \frac{\partial \bar{b}_{\theta}}{\partial \bar{r}}=0, \\
\bar{r}=\frac{r_{o}}{a}=\bar{r}_{o}, & \bar{u}_{\theta}=\frac{u_{o}}{u_{o}}=1, & \bar{b}_{\theta}=0 .
\end{array}
$$

\section{Solutions}

The solutions to the set of governing partial differential equations are strongly dependent on the appropriate boundary conditions associated with the problem being considered. Solving equation (5) simultaneously subjected to the boundary conditions ((6a) and (6b)), ((7a) and (7b)), or ((8a) and $(8 b))$, one can obtain the expressions for the profiles for the dimensionless velocity and the dimensionless induced magnetic field.

(a) If both the inner and outer cylinders are perfectly insulated, the expressions for the velocity $(u)$ and induced magnetic field $(b)$ profiles, after dropping the bar for brevity, can be obtained by solving (5) simultaneously subjected to boundary conditions ((6a) and (6b)). These profiles read

$$
\begin{aligned}
u= & C_{1} r^{m}+C_{2} r^{-m}+C_{3} \frac{\mathrm{Ha}}{m^{2}-1} r, \\
b= & -C_{1} \frac{\mathrm{Ha}}{m+1} r^{m}+C_{2} \frac{\mathrm{Ha}}{m-1} r^{-m} \\
& -\frac{C_{3}}{2}\left(\frac{\mathrm{Ha}}{m^{2}-1}-1\right) r+C_{4} r^{-1},
\end{aligned}
$$

where

$$
\begin{gathered}
m=\sqrt{\mathrm{Ha}^{2}+\frac{1}{\mathrm{Da}}+1,} \\
\omega=\frac{u_{i}}{u_{o}}, \\
\phi=\frac{r_{i}}{r_{o}}, \\
C_{1}=\frac{(\omega-\phi) f_{1}-\left(\phi^{-m}-\phi\right)\left(1-\phi^{2}\right) g}{\left(\phi^{m}-\phi\right) f_{1}-\left(\phi^{-m}-\phi\right) f_{2}}, \\
C_{2}=\frac{-(\omega-\phi) f_{2}+\left(\phi^{m}-\phi\right)\left(1-\phi^{2}\right) g}{\left(\phi^{m}-\phi\right) f_{1}-\left(\phi^{-m}-\phi\right) f_{2}}, \\
\frac{m^{2}-1}{\mathrm{Ha}} \\
\times \frac{(10 \mathrm{~b})}{\left(\phi^{m}-\omega\right) f_{1}+\left(\omega-\phi^{-m}\right) f_{2}+\left(\phi^{-m}-\phi^{m}\right)\left(1-\phi^{2}\right) g}
\end{gathered}
$$

$$
C_{4}=\frac{\mathrm{Ha}}{m+1} C_{1} \phi^{m+1}-\frac{\mathrm{Ha}}{m-1} C_{2} \phi^{-m+1}+\left(1-C_{1}-C_{2}\right) \phi^{2} g,
$$

$$
\begin{gathered}
f_{1}=\frac{\mathrm{Ha}}{m-1}\left(1-\phi^{-m+1}\right)+\left(1-\phi^{2}\right) g \\
f_{2}=-\frac{\mathrm{Ha}}{m+1}\left(1-\phi^{m+1}\right)+\left(1-\phi^{2}\right) g, \\
g=\frac{\mathrm{Ha}^{2}-m^{2}+1}{2 \mathrm{Ha}} .
\end{gathered}
$$

(b) If the inner cylinder is perfectly insulated but the outer cylinder is perfectly conducting, the velocity and induced magnetic field profiles associated with boundary conditions $((7 \mathrm{a})$ and $(7 \mathrm{~b}))$ are also given by ((9a) and (9b)), but the coefficients $C_{1}$ through $C_{4}$ are redefined as below:

$$
\begin{gathered}
C_{1}=\frac{(\omega-\phi) f_{3}-\left(\phi^{-m}-\phi\right)\left(\phi^{2}+1\right) g}{\left(\phi^{m}-\phi\right) f_{3}+\left(\phi^{-m}-\phi\right) f_{4}} \\
C_{2}=\frac{(\omega-\phi) f_{4}+\left(\phi^{m}-\phi\right)\left(\phi^{2}+1\right) g}{\left(\phi^{m}-\phi\right) f_{3}+\left(\phi^{-m}-\phi\right) f_{4}}
\end{gathered}
$$

$$
\begin{aligned}
C_{3}= & \frac{m^{2}-1}{\mathrm{Ha}} \\
& \times \frac{\left(\phi^{m}-\omega\right) f_{3}-\left(\omega-\phi^{-m}\right) f_{4}-\left(\phi^{m}-\phi^{-m}\right)\left(\phi^{2}+1\right) g}{\left(\phi^{m}-\phi\right) f_{3}+\left(\phi^{-m}-\phi\right) f_{4}},
\end{aligned}
$$




$$
\begin{gathered}
C_{4}=\frac{\mathrm{Ha}}{m+1} C_{1} \phi^{m+1}-\frac{\mathrm{Ha}}{m-1} C_{2} \phi^{-m+1}+\left(1-C_{1}-C_{2}\right) \phi^{2} g \\
f_{3}=\frac{\mathrm{Ha}}{m-1}\left(-m+\phi^{-m+1}\right)+\left(1+\phi^{2}\right) g, \\
f_{4}=\frac{\mathrm{Ha}}{m+1}\left(m+\phi^{m+1}\right)-\left(1+\phi^{2}\right) g,
\end{gathered}
$$

where $g$ is exactly the same as defined in (10j).

(c) If the outer cylinder is perfectly insulated but the inner cylinder is perfectly conducting, the velocity and induced magnetic field profiles associated with boundary conditions $((8 \mathrm{a})$ and $(8 \mathrm{~b}))$ are also given by $((9 \mathrm{a})$ and $(9 \mathrm{~b}))$ whose coefficients $C_{1}$ through $C_{4}$ are once again redefined as below:

$$
\begin{gathered}
C_{1}=\frac{(\varrho-\phi) f_{5}-\left(\phi^{-m}-\phi\right)\left(\phi^{2}+1\right) g}{\left(\phi^{m}-\phi\right) f_{5}+\left(\phi^{-m}-\phi\right) f_{6}}, \quad \text { (12a) } \\
C_{2}=\frac{(\varpi-\phi) f_{6}+\left(\phi^{m}-\phi\right)\left(\phi^{2}+1\right) g}{\left(\phi^{m}-\phi\right) f_{5}+\left(\phi^{-m}-\phi\right) f_{6}}, \quad \text { (12b) } \\
C_{3}=-\frac{1-m^{2}}{\mathrm{Ha}} \\
\times \frac{\left(\phi^{m}-\omega\right) f_{5}-\left(\emptyset-\phi^{-m}\right) f_{6}-\left(\phi^{m}-\phi^{-m}\right)\left(\phi^{2}+1\right) g}{\left(\phi^{m}-\phi\right) f_{5}+\left(\phi^{-m}-\phi\right) f_{6}}, \quad \text { (12c) } \\
C_{4}=-\frac{\mathrm{Ha}}{m+1} C_{1} m \phi^{m+1}-\frac{\mathrm{Ha}}{m-1} C_{2} m \phi^{-m+1} \\
-\left(1-C_{1}-C_{2}\right) \phi^{2} g, \\
f_{5}=\frac{\mathrm{Ha}}{m-1}\left(1-m \phi^{-m+1}\right)+\left(1+\phi^{2}\right) g, \\
f_{6}=\frac{\mathrm{Ha}}{m+1}\left(1+m \phi^{m+1}\right)-\left(1+\phi^{2}\right) g,
\end{gathered}
$$

where $g$ is also identical to the one defined (10j).

\section{Results and Discussion}

To validate the solutions presented in the previous section, these solutions have been compared to the analytical solution for a flow between a set of rotating cylinders [36]. It is remarkable to point out that there appeared a singularity point associated with $\mathrm{Ha}=0$ for all the solutions obtained here. To avoid the singularity, Ha was assigned $10^{-3}$ which physically indicates the presence of an extremely weak external magnetic field. Also, Da was assigned $10^{3}$ to suppress the tortuous effect associated with the porous annulus. Figure 2 shows the three solutions obtained in this work. Solutions (a), (b), and (c) correspond to the cases where both the

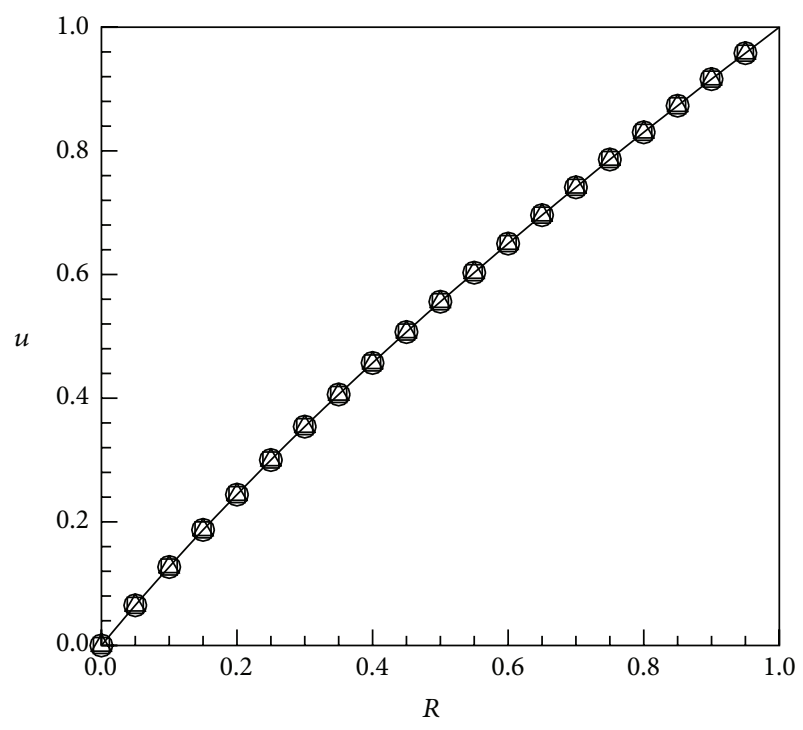

FIGURE 2: Validation of present solution with $\mathrm{Ha}=10^{-3}$ and $\mathrm{Da}=$ $10^{3}$. -Currie [36], $\square$ Solution (a), $\triangle$ Solution (b), and O Solution (c).

inner and outer cylinders are perfectly insulated, the case where the inner cylinder is perfectly insulated but the outer cylinder is perfectly conducting, and the case where the outer cylinder is perfectly insulated but the inner cylinder is perfectly conducting. All these solutions are identical as evidently shown in the figure.

For demonstration, present study is limited to the settings of $\bar{r}_{i}=1, \bar{r}_{o}=2, \bar{u}_{i}=0$, and $\bar{u}_{o}=1$ although arbitrary values are acceptable as long as $\bar{u}_{o} \neq 0$. Therefore, in the figures that follow, $R=1$ represents the locations of the inner cylinder while $R=2$ the outer cylinder. Figure 3 shows the velocity and induced magnetic field profiles between the perfectly insulated inner and outer cylinders for $\mathrm{Da}=$ $10^{-2}$ corresponding to different values of Ha. As shown in Figure 3(a), the effect of porous media is so significant that the flow drag that the porous matrix imposes causes the velocity to collapse in the vicinity of the stationary inner cylinder. As Ha increases, the Lorentz force induced within the fluid provides additional momentum to the fluid elements close to the inner cylinder and thus accelerates the fluid elements there. At the same time, the fluid elements closer to the rotating outer cylinder tends to slightly slow down due to the presence of a stronger Lorentz force in conjunction with a greater value of permeability (i.e., a weaker flow resistance). The profiles of the induced magnetic field shown in Figure 3(b) prove that its amplitude increases with $\mathrm{Ha}$ which is an indication for the strength of the externally applied magnetic field. A maximum amount of magnetic field induced is found within the region $1.6<R<1.8$. This phenomenon is closely related to the region where the change in velocity gradient is the most significant. Since both the inner and outer cylinders are perfectly insulated, no electric current is allowed to flow within these solid cylinders. The induced magnetic field close to these two cylindrical walls is thus very small in accordance with Ampere's Law. 


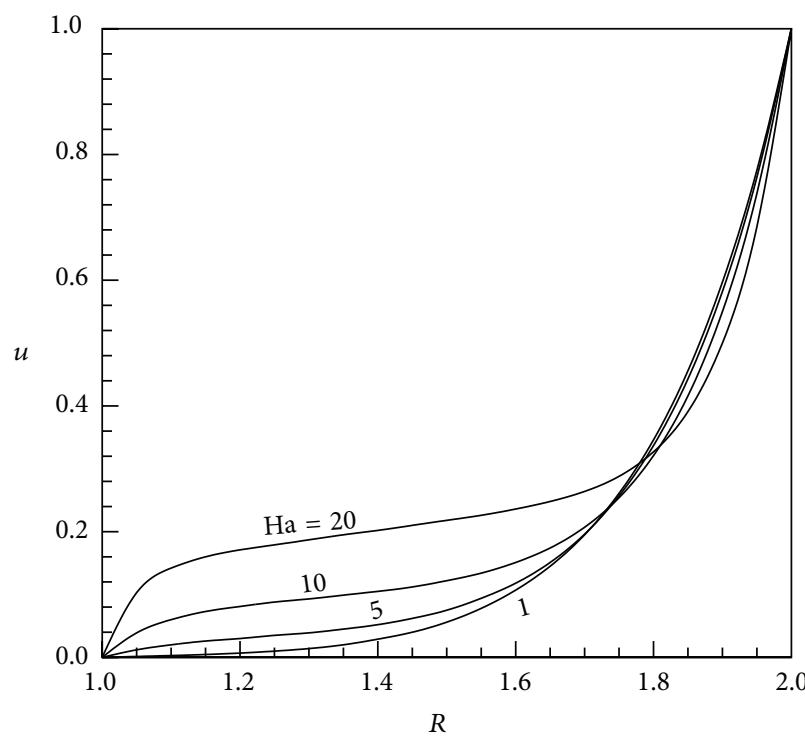

(a)

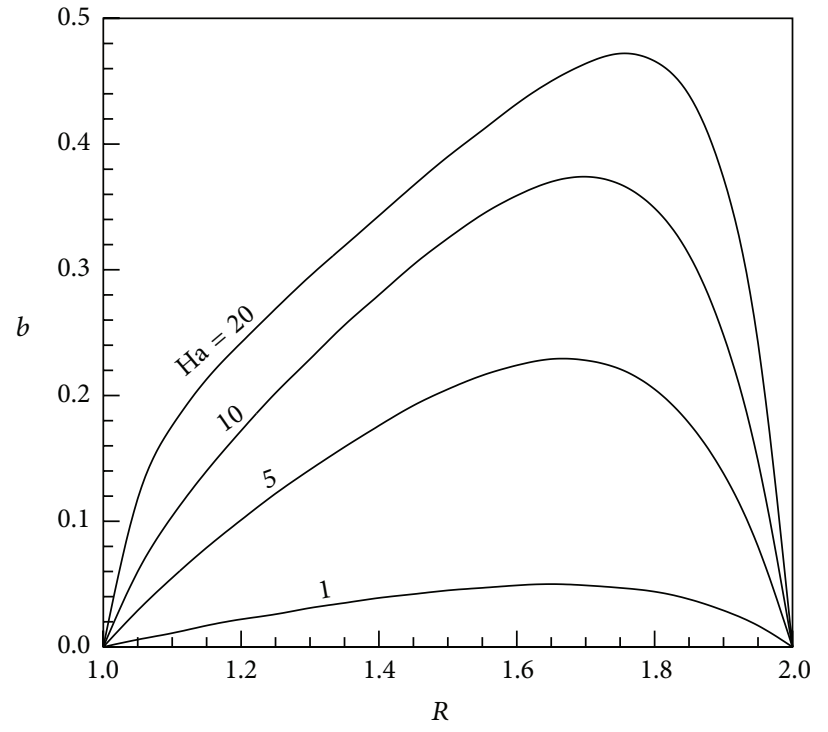

(b)

Figure 3: Various profiles for $\mathrm{Da}=10^{-2}$ and different values of Ha when both cylinders are insulated: (a) velocity and (b) induced magnetic field.

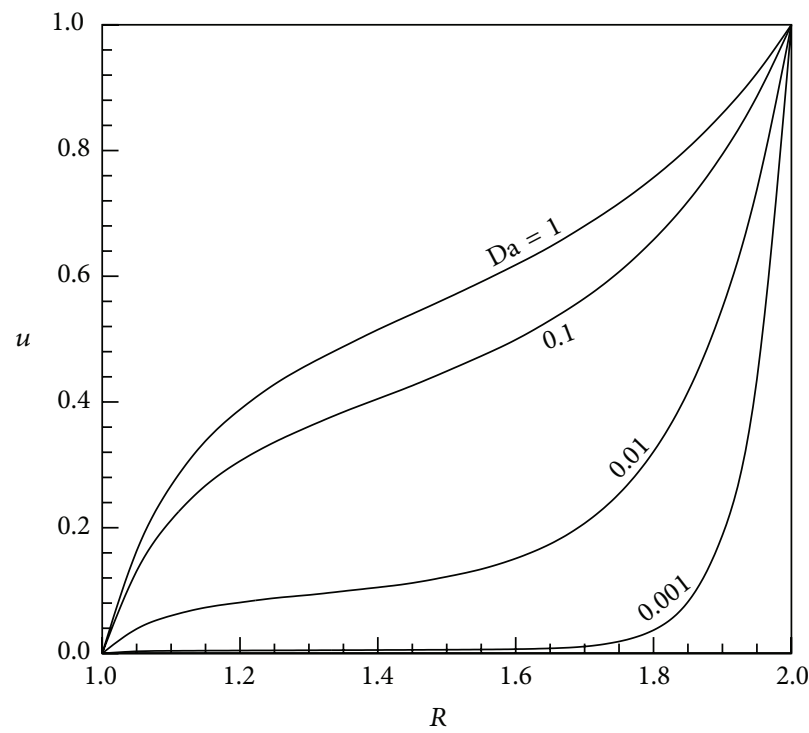

(a)

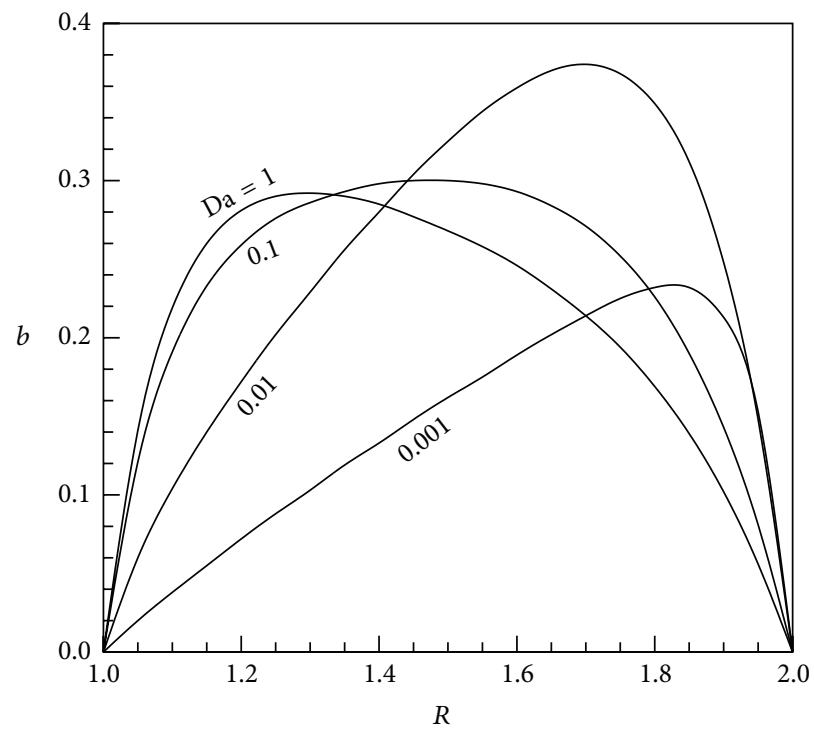

(b)

Figure 4: Various profiles for $\mathrm{Ha}=10$ and different values of $\mathrm{Da}$ when both cylinders are insulated: (a) velocity and (b) induced magnetic field.

The effects of $\mathrm{Da}$ are investigated in Figure 4. When $\mathrm{Ha}=$ 10 , it is apparent that the motion of the fluid is significantly influenced by the permeability of the porous annulus. As Da gradually reduces, the additional flow resistance that the porous structure offers increases. This leads to a reduction in flow rate across the gap, as apparently shown in Figure 4(a). It is remarkable to emphasize that the velocity profiles at $\mathrm{Da}=$ $10^{-4}$ reduce to zero except in the vicinity of the rotating inner cylinder, that is, the Hartmann layer. When $\mathrm{Da} \leq 10^{-3}$, the induced Lorentz force even for an external magnetic field of
$\mathrm{Ha}=10$ is still relatively too weak to assist magnetic fluid to overcome the flow resistance due to the presence of porous structure. Hence, no more than $25 \%$ of the gap contributes to the overall mass transfer if $\mathrm{Da}<10^{-3}$ because the fluid elements outside the Hartmann layer are literally motionless. A further reduction in Da would eventually retard the flow resulting in a much narrower Hartmann layer right next to the rotating outer cylinder. The corresponding distribution of induce magnetic field is shown in Figure 4(b). The maximum induced magnetic field strength for different $\mathrm{Da}$ does not 


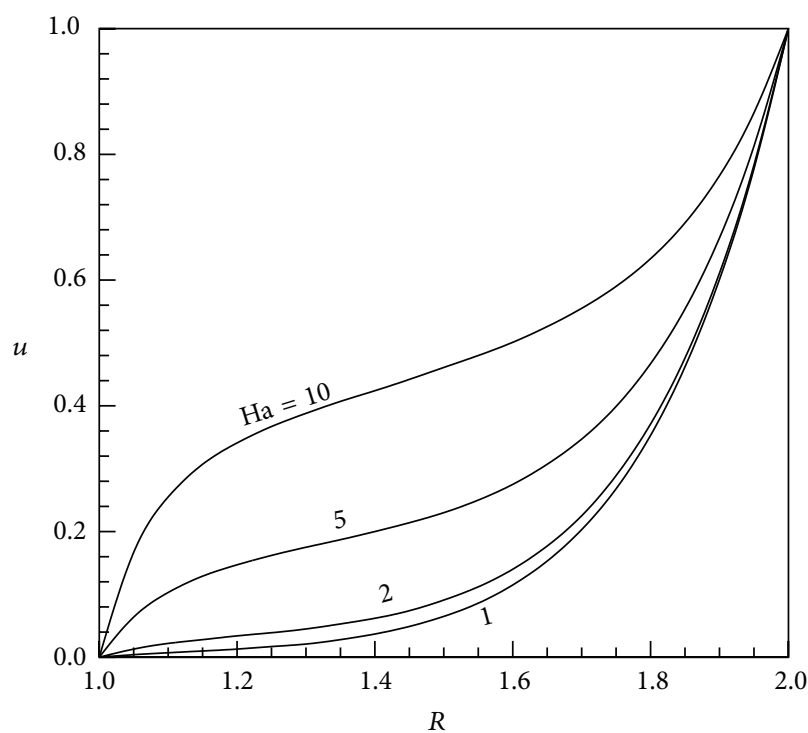

(a)

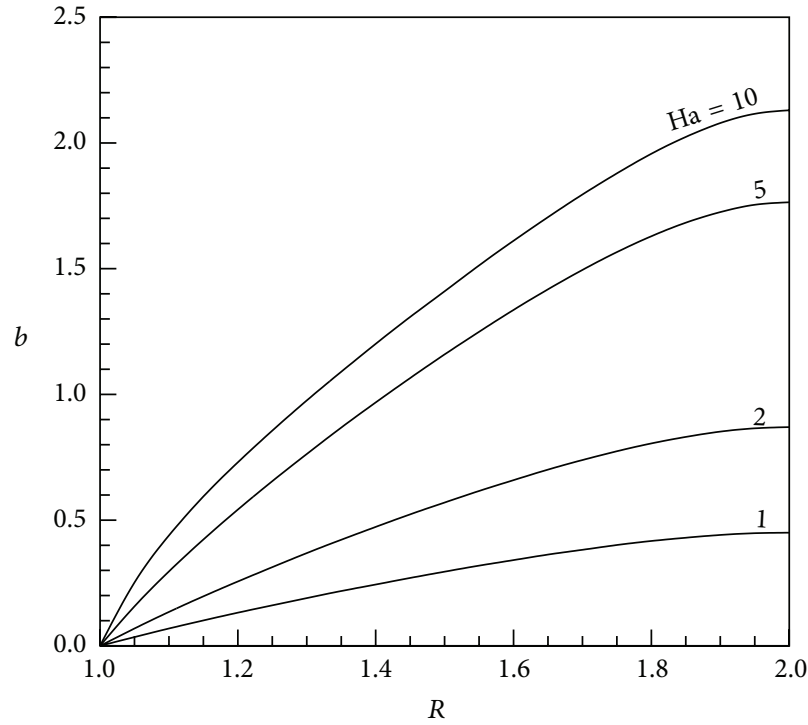

(b)

FIGURE 5: Various profiles for $\mathrm{Da}=10^{-2}$ and different values of $\mathrm{Ha}$ when the inner cylinder is perfectly insulated while the outer one is perfectly conducting: (a) velocity and (b) induced magnetic field.

seem to follow any specific pattern. When Da reduces from $10^{0}$ to $10^{-2}$, it increases. However, a further reduction in $\mathrm{Da}$ leads to its dramatic reduction. In fact, there is a reason behind the aforementioned phenomenon. The increase in magnetic induction for $\mathrm{Da}>10^{-2}$ is attributed to the increase in velocity gradient in the outer region of the magnetic fluid flow. If $\mathrm{Da}<10^{-2}$, the conducting fluid is mostly motionless as shown in Figure 4(a), it is this lack of momentum that causes the decrease in induced magnetic field strength. Despite the presence of this motionless fluid region, the velocity gradient across the fluid layer next to the outer cylinder contributes to the maximum magnetic field strength induced at $R \approx 1.85$.

At $\mathrm{Da}=10^{-2}$, the magnitude of the velocity associated with a perfectly conducting outer cylinder that allows electric currents to flow through is apparently greater than those between a pair of insulated cylinders, through the comparison made between Figures 3(a) and 5(a). Although these velocity profiles have some resemblances at relatively low $\mathrm{Ha}$, the velocity profiles are quite distinctive at high values of Ha. Unlike those profiles shown in Figure 3(a), the velocity gradient on the rotating perfectly conducting outer cylindrical wall decreases with $\mathrm{Ha}$. This implies that the torque requirement for the outer cylinder actually lessens if more magnetic energy is applied to the annulus. Even though the velocity profiles for $\mathrm{Ha}=1$ and $\mathrm{Ha}=2$ are very similar to each other, the profile for induced magnetic field for $\mathrm{Ha}=2$ is obviously greater than that for $\mathrm{Ha}=1$, as shown in Figure 5(b). In this figure, it is observed that the strength of the induced magnetic field increases with $\mathrm{Ha}$. Not only so, the maximum magnetic strength occurs at the perfectly conducting outer cylinder. This finite value of induced magnetic field on the outer cylindrical wall has no doubt suggested that there exists an induced electric current within the solid outer cylinder.
Because the outer cylinder is perfectly conductive, part of the electric current induced by the MHD flow will be able to bypass through the outer cylinder. Because of this additional flow of electrons within the outer cylinder, it indirectly helps the induction of additional magnetic field in the flow region next to the outer cylinder.

Figure 6 demonstrates the effect of permeability on the velocity and induced magnetic field of the magnetic fluid. At a $\mathrm{Ha}$ of 10, the Lorentz force induced is strong enough to accelerate the magnetic fluid and thus the velocity profiles are very similar when $\mathrm{Da} \geq 0.1$. However, if $\mathrm{Da}$ is further decreased, the effect of flow resistance due to porous media begins to dominate the effect of Lorentz force. For this reason, the overall velocity between the cylinders reduces. However, it is observed that even when $\mathrm{Da}=10^{-3}$, there is a finite velocity component throughout the gap between the inner and outer cylinders. Apparently, the conductivity of the outer cylinder has significantly improved the Lorentz force and thus maintained a minimum flow rate across the gap. However, it is remarkable to point out that this minimum flow rate will eventually reduce to nearly zero if $\mathrm{Da}=10^{-5}$ with a very thin Hartmann layer. As shown in Figure 6(b), the induced magnetic field is the weakest when $\mathrm{Da}=1$. In this case, there exists a maximum magnetic strength at $R \approx$ 1.2. As Da decreases, the amount of magnetic field induced increases significantly. For instance, when $\mathrm{Da}=10^{-3}$, the strength of the magnetic field induced can be as high as 4.8. This clearly implies that the conductivity of the cylinder plays an important role in magnetic field induction.

Figure 7 shows the profiles of velocity and induced magnetic field at fixed value of $\mathrm{Da}=10^{-2}$. Since the fluid elements in the outer half of the annulus next to the outer cylinder experience a stronger Lorentz force than those in the 


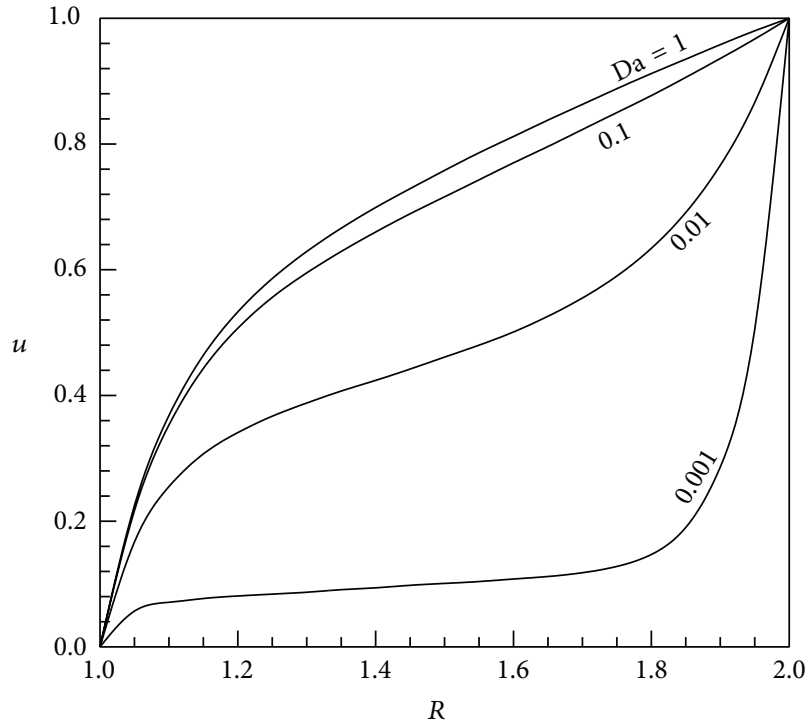

(a)

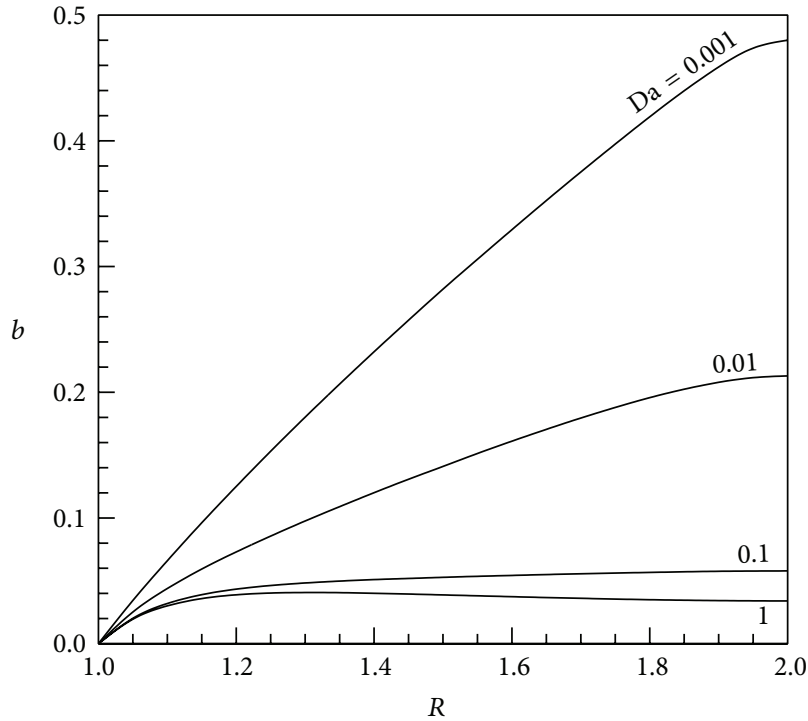

(b)

Figure 6: Various profiles for $\mathrm{Ha}=10$ and different values of $\mathrm{Da}$ when the inner cylinder is perfectly insulated while the outer one is perfectly conducting: (a) velocity and (b) induced magnetic field.

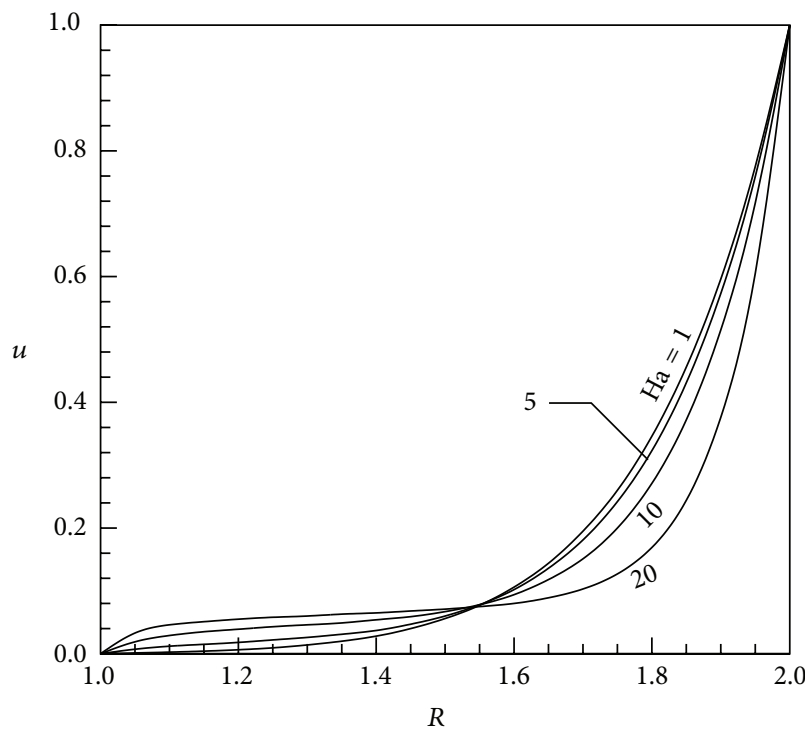

(a)

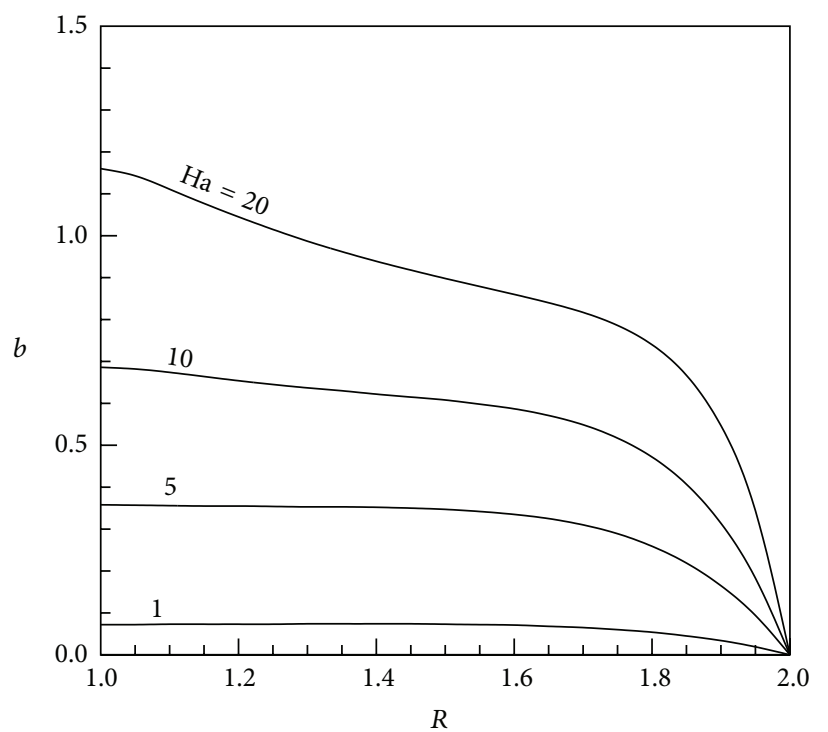

(b)

FIGURE 7: Various profiles for $\mathrm{Da}=10^{-2}$ and different values of Ha when the outer cylinder is perfectly insulated while the inner one is perfectly conducting: (a) velocity and (b) induced magnetic field.

other half of the annulus, the velocity gradients at both the inner and outer cylinders increase with $\mathrm{Ha}$. It is also found that there exists an invariant point associated with the velocity component at $R \approx 1.55$. Furthermore, the flow rate across the gap decreases with Ha because the drop in velocity magnitude between the velocity invariant point and the outer cylinder is always greater than that between the invariant point and the inner cylinder. Accordingly, the average velocity within the gap between a perfectly conducting nonmoving inner cylinder and a perfectly insulating rotating outer cylinder is always greater than other conditions discussed before. When $\mathrm{Ha} \leq 5$, the induced magnetic field away from the inner cylinder remains almost unchanged in the radial direction until it is closed to the outer cylinder where its strength begins to decay sharply. Therefore, the maximum induced magnetic field generally takes place on the inner cylindrical wall.

Figure 8 demonstrates the profiles of velocity and induced magnetic field when $\mathrm{Ha}=10$. The boundary conditions are identical to the cases investigated in Figure 7. If $D a$ decreases from $10^{0}$ to $10^{-1}$, it means the permeability of the porous 


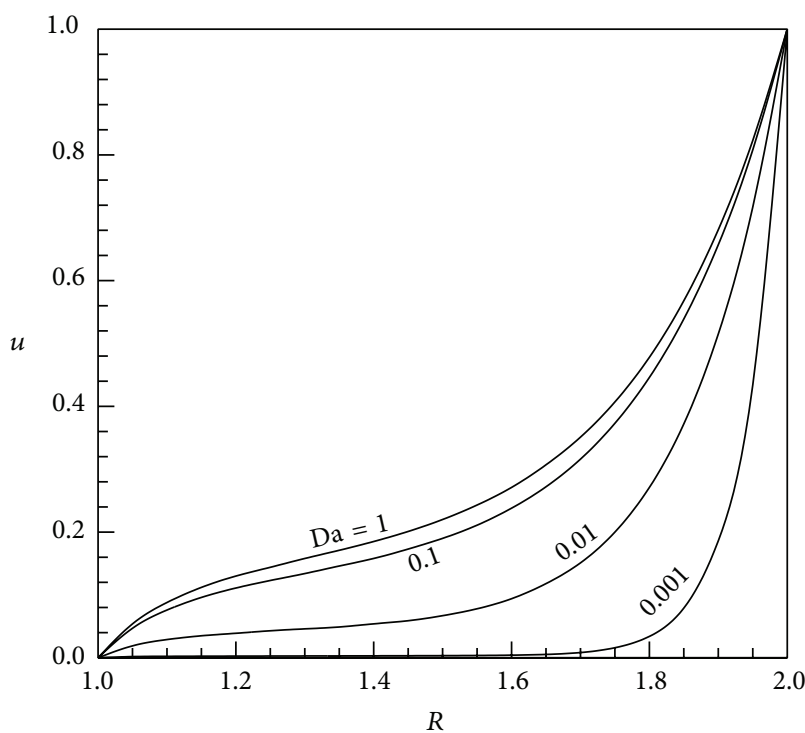

(a)

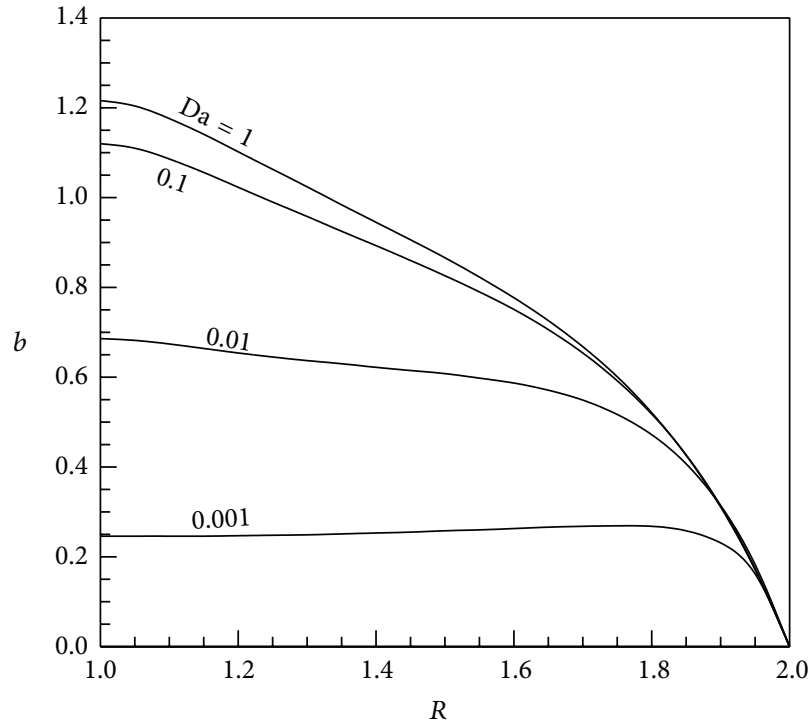

(b)

FIGURE 8: Various profiles for $\mathrm{Ha}=10$ and different values of $\mathrm{Da}$ when the outer cylinder is perfectly insulated while the inner one is perfectly conducting: (a) velocity and (b) induced magnetic field.

annulus decreases. When this happens, the fluid elements are required to overcome a greater flow resistance before it can penetrate the porous annulus. Thus, their velocity everywhere within the gap lowers. When Da reduces to $10^{-2}$, the transfer of the momentum from the outer cylinder has been apparently suppressed by the additional flow resistance caused by the less porous annulus. The effect of flow suppression at $\mathrm{Da}=10^{-3}$ is so significant that most of the flow within the gap is nearly motionless. This supports the fact that the flow resistance effect has become the primary factor influencing the flow nature. Because of the reduction in velocity, the magnitude of magnetic field induced decreases accordingly, as clearly displayed in Figure 8(b). When Da decreases gradually, the strength of magnetic field induced in the vicinity of the inner cylinder is the greatest unless $\mathrm{Da}$ is small enough. Not only so, the induced magnetic field on the inner cylinder drops tremendously as Da decreases.

\section{Conclusions}

A set of simplified governing equations for a steady cylindrical magnetic Couette flow in a porous annulus between two cylinders under the influence of a radial magnetic field has been established and their analytical solutions have been obtained. The main purpose of this paper is to study how the strength of the externally applied magnetic field; the permeability of the porous annulus; and the conductivity of the inner and outer cylinders affect the flow behaviors and magnetic field induction within the magnetic fluid. The following conclusions were obtained.

(a) A closed form solution is available if the permeability of the porous annulus increases with its radial coordinates; that is, $K_{p}=\alpha r^{2}$, where $\alpha$ is a constant. (b) Both the Lorentz force and flow resistance due to the porous structure tend to unify the flow within the gap. The former accelerates or decelerates the magnetic fluid depending on its location, whereas the latter always decelerates the flow.

(c) The strength of the induced magnetic field increases with $\mathrm{Ha}$ because of the increase in induced electric current. However, this strength decreases with Da because of the reduction of flow momentum that transfers within the porous annulus.

(d) The conductivity of the cylinders is highly influential on the velocity and induced magnetic field profiles.

\section{Nomenclature}

$a$ : Gap between inner and outer cylinder, $m$

$\vec{B}$ : Magnetic flux density, Tesla

$B_{0}$ : External magnetic field density, Tesla

$b_{0}$ : An arbitrary magnetic scale used in normalization, Tesla

$b_{\theta}$ : Angular component of induced magnetic field density, Tesla

Da: Darcy number, -

$f$ : $\quad$ Lorentz force, $\mathrm{N}$

Ha: Hartmann number, -

$j: \quad$ Induced current density, $\mathrm{A} / \mathrm{m}^{2}$

$K_{p}$ : Permeability of porous media, $\mathrm{m}^{2}$

$r:$ Radial coordinate, $\mathrm{m}$

$\vec{V}:$ Fluid velocity, $\mathrm{m} / \mathrm{s}$

$u_{0}$ : An arbitrary velocity scale used in normalization, $\mathrm{m} / \mathrm{s}$

$u_{\theta}$ : Fluid angular velocity component, $\mathrm{m} / \mathrm{s}$. 


\section{Greek}

$\varepsilon$ : Magnetic permittivity of material, $\mathrm{F} / \mathrm{m}$

$\mu$ : Magnetic permeability of material, $\mathrm{H} / \mathrm{m}$

$v$ : Fluid kinematic viscosity, $\mathrm{m}^{2} / \mathrm{s}$

$\theta$ : Angular coordinate, -

$\rho$ : Fluid density, $\mathrm{kg} / \mathrm{m}^{3}$

$\sigma$ : Electric conductivity, $1 / \Omega \cdot \mathrm{m}$.

\section{Subscripts}

$i$ : Inner cylinder

$o$ : Outer cylinder

$z$ : Axial direction

$\theta$ : Angular direction.

\section{Conflict of Interests}

The authors declare that there is no conflict of interests regarding the publication of this paper.

\section{References}

[1] D. T. Swift-Hook and J. K. Wright, "The constant-Mach-number MHD generator," Journal of Fluid Mechanics, vol. 15, pp. 97-110, 1963.

[2] S. O. Macheret, M. N. Shneider, and R. B. Miles, "Potential performance of supersonic MHD power generators," in Proceedings of the AIAA 39th Aerospace Sciences Meeting \& Exhibition, AIAA-2001-0795, Reno, Nev, USA, January 2001.

[3] B. F. Howell Jr., Introduction To Geophysics, McGraw Hill, New York, NY, USA, 1959.

[4] A. A. Kaufman and G. V. Keller, The Magnetotelluric Sounding Method, Elsevier, New York, NY, USA, 1981.

[5] F. Troyon, R. Gruber, H. Saurenmann, S. Semenzato, and S. Succi, "MHD-Limits to plasma confinement," in Proceedings of the 11th European Conference on Controlled Fusion and Plasma Physics, Aachen, Germany, September 1983.

[6] Y. Aoki, T. Seidou, and N. Ohtomo, "Measurement of the timedependent temperature variation of combustion MHD plasma," Japanese Journal of Applied Physics, vol. 23, no. 12, pp. 1628-1633, 1984.

[7] J. E. Borovsky, R. C. Elphic, H. O. Funsten, and M. F. Thomsen, "The Earth's plasma sheet as a laboratory for flow turbulence in high- $\beta$ MHD," Journal of Plasma Physics, vol. 57, no. 1, pp. 1-34, 1997.

[8] Y.-K. Wu, "Magnetohydrodynamic boundary layer control with suction or injection," Journal of Applied Physics, vol. 44, no. 5, pp. 2166-2171, 1973.

[9] D. M. Bushnell and C. B. McGinley, "Turbulence control in wall flows," Annual Review of Fluid Mechanics, vol. 21, pp. 1-20, 1989.

[10] M. Nishihara, N. Jiang, J. W. Rich, W. R. Lempert, I. V. Adamovich, and S. Gogineni, "Low-temperature supersonic boundary layer control using repetitively pulsed magnetohydrodynamic forcing," Physics of Fluids, vol. 17, no. 10, Article ID 106102, 2005.

[11] R. Moreau, Magnetohydrodynamics, Kluwer Academic Publishers, Boston, Mass, USA, 1990.

[12] J. A. Shercliff, "Steady motion of conducting fluids in pipes under transverse magnetic fields," Proceedings of the Cambridge Philosophical Society, vol. 49, pp. 136-144, 1953.
[13] J. A. Shercliff, "The flow of conducting fluids in circular pipes under transverse magnetic fields," Journal of Fluid Mechanics, vol. 1, pp. 644-666, 1956.

[14] J. C. R. Hunt and K. Stewartson, "Magnetohydrodynamic flow in rectangular ducts. II," Journal of Fluid Mechanics, vol. 23, part 3, pp. 563-581, 1965.

[15] S. C. Gupta and B. Singh, "Unsteady magnetohydrodynamic flow in a circular pipe under a transverse magnetic field," Physics of Fluids, vol. 13, no. 2, pp. 346-352, 1970.

[16] S. C. Gupta, "Unsteady magnetohydrodynamic flow in a rectangular channel under transverse magnetic field," Indian Journal of Pure and Applied Mathematics, vol. 3, no. 6, pp. 1038-1047, 1972.

[17] W. H. Heiser and J. A. Shercliff, "A simple demonstration of the Hartmann layer," Journal of Fluid Mechanics, vol. 22, part 4, pp. 701-707, 1965.

[18] S. Y. Molokov and J. E. Allen, "Theory of the Heiser and Shercliff experiment. II: MHD flow between two cylinders in a strong radial magnetic field," Journal of Physics D: Applied Physics, vol. 25, no. 6, pp. 933-937, 1992.

[19] C. H. Tsai, J. S. Kuo, and J. C. Leong, "A theoretical analysis of MHD Couette flow in a thin cylindrical annulus," Advanced Science Letters, vol. 12, pp. 120-124, 2012.

[20] J.-S. Kuo and J. C. Leong, "Analysis of a conducting fluid in a thin annulus with rotating insulated walls under radial magnetic effect," Applied Mathematical Modelling, vol. 37, no. 5, pp. 3021-3035, 2013.

[21] T. Hayat, M. Khan, and M. Ayub, "Couette and Poiseuille flows of an Oldroyd 6-constant fluid with magnetic field," Journal of Mathematical Analysis and Applications, vol. 298, no. 1, pp. 225244, 2004.

[22] E. P. Velikhov, A. A. Ivanov, S. V. Zakharov, V. S. Zakharov, A. O. Livadny, and K. S. Serebrennikov, "Equilibrium of current driven rotating liquid metal," Physics Letters A, vol. 358, no. 3, pp. 216-221, 2006.

[23] W. Tan and T. Masuoka, "Stokes' first problem for an OldroydB fluid in a porous half space," Physics of Fluids, vol. 17, no. 2, Article ID 023101, 2005.

[24] F. Salah, Z. Abdul Aziz, and D. L. C. Ching, "New exact solutions for MHD transient rotating flow of a second-grade fluid in a porous medium," Journal of Applied Mathematics, vol. 2011, Article ID 823034, 8 pages, 2011.

[25] I. Khan, M. Imran, and K. Fakhar, "New exact solutions for an Oldroyd-B fluid in a porous medium," International Journal of Mathematics and Mathematical Sciences, vol. 2011, Article ID 408132, 12 pages, 2011.

[26] M. Khan, M. Saleem, C. Fetecau, and T. Hayat, "Transient oscillatory and constantly accelerated non-Newtonian flow in a porous medium," International Journal of Non-Linear Mechanics, vol. 42, no. 10, pp. 1224-1239, 2007.

[27] M. Khan, E. Naheed, C. Fetecau, and T. Hayat, "Exact solutions of starting flows for second grade fluid in a porous medium," International Journal of Non-Linear Mechanics, vol. 43, no. 9, pp. 868-879, 2008.

[28] T. Hayat, N. Ahmed, and M. Sajid, "Analytic solution for MHD flow of a third order fluid in a porous channel," Journal of Computational and Applied Mathematics, vol. 214, no. 2, pp. 572-582, 2008.

[29] G. S. Seth, M. S. Ansari, and R. Nandkeolyar, "Unsteady hydromagnetic couette flow within a porous channel," Tamkang Journal of Science and Engineering, vol. 14, no. 1, pp. 7-14, 2011. 
[30] O. A. Bég, S. K. Ghosh, and M. Narahari, "Mathematical modeling of oscillatory MHD couette flow in a rotating highly permeable medium permeated by an oblique magnetic field," Chemical Engineering Communications, vol. 198, no. 2, pp. 235254, 2011.

[31] J. V. Ramana Murthy, N. K. Bahali, and D. Srinivasacharya, "Unsteady flow of micropolar fluid through a circular pipe under a transverse magnetic field with suction/injection," Selçuk Journal of Applied Mathematics, vol. 11, no. 2, pp. 13-25, 2010.

[32] M. Khan, T. Hayat, and S. Asghar, "Exact solution for MHD flow of a generalized Oldroyd-B fluid with modified Darcy's law," International Journal of Engineering Science, vol. 44, no. 5-6, pp. 333-339, 2006.

[33] S. Srinivas and R. Muthuraj, "MHD flow with slip effects and temperature-dependent heat source in a vertical wavy porous space," Chemical Engineering Communications, vol. 197, no. 11, pp. 1387-1403, 2010.

[34] O. D. Makinde, O. A. Bég, and H. S. Takhar, "Magnetohydrodynamic viscous flow in a rotating porous medium cylindrical annulus with an applied radial magnetic field," International Journal of Applied Mathematics and Mechanics, vol. 5, pp. 6881, 2009.

[35] P. A. Davidson and A. Thess, Magnetohydrodynamics, Springer, New York, NY, USA, 2002.

[36] I. G. Currie, Fundamental Mechanics of Fluids, McGraw-Hill, Taipei, Taiwan, 2nd edition, 2004. 


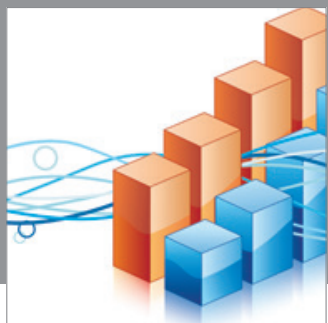

Advances in

Operations Research

mansans

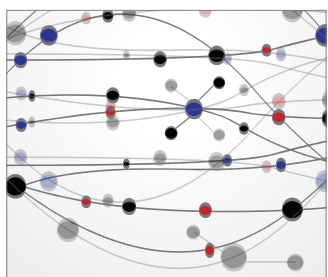

The Scientific World Journal
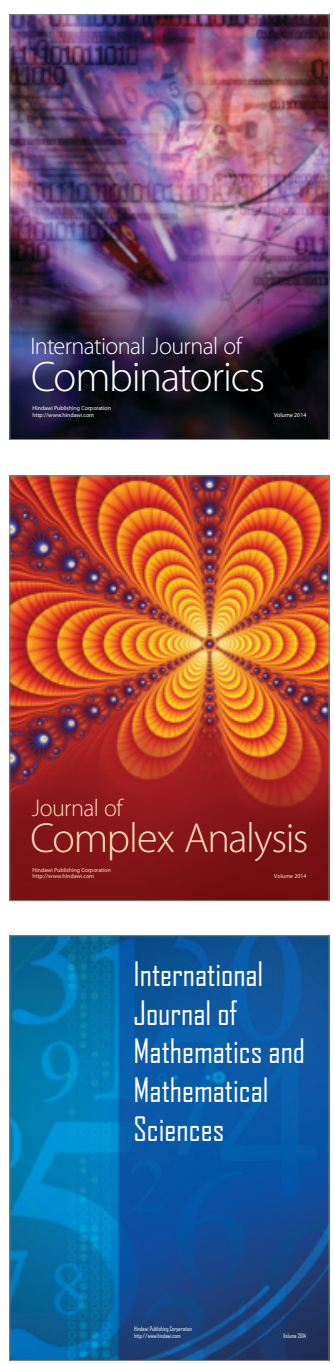
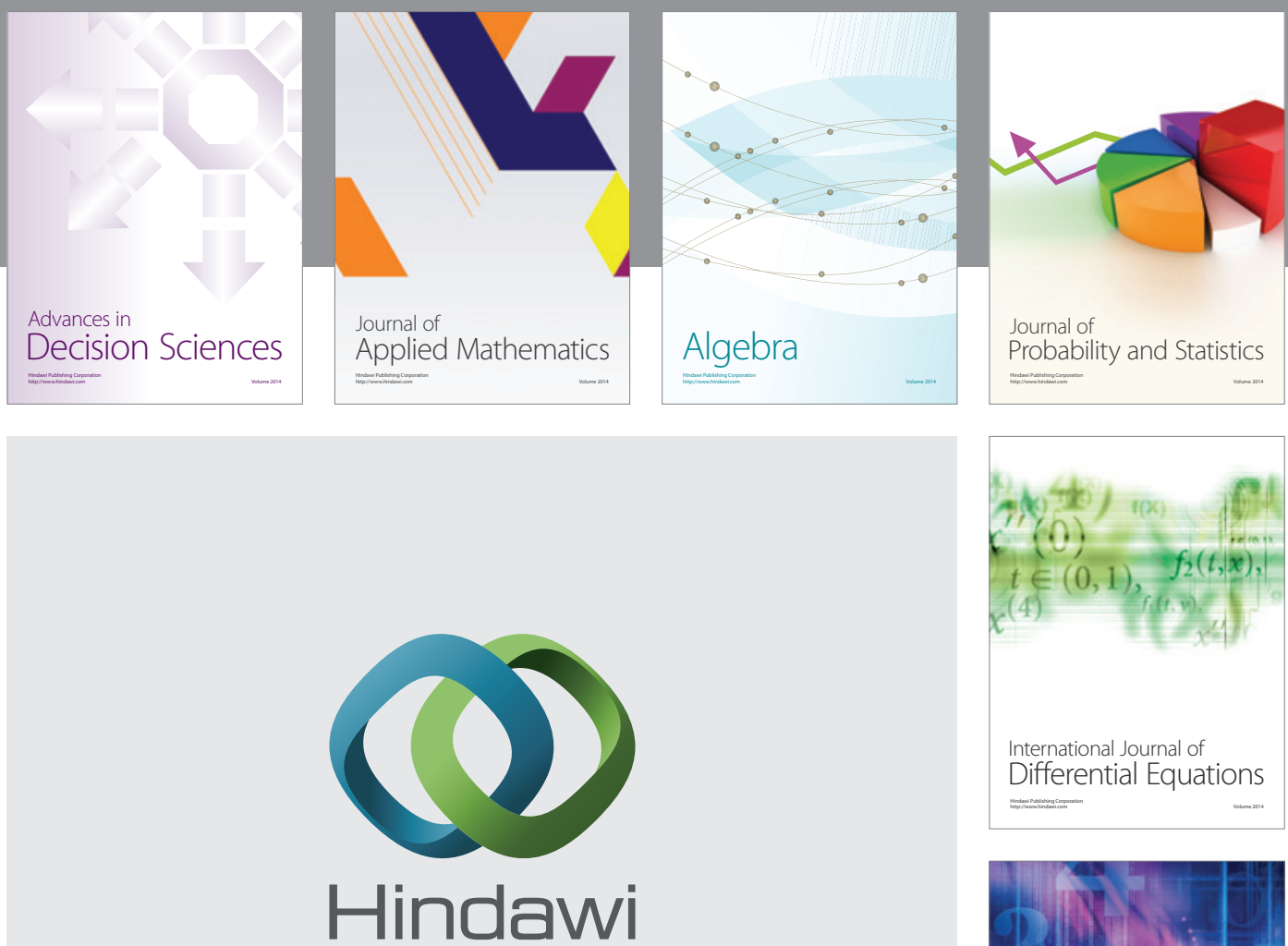

Submit your manuscripts at http://www.hindawi.com
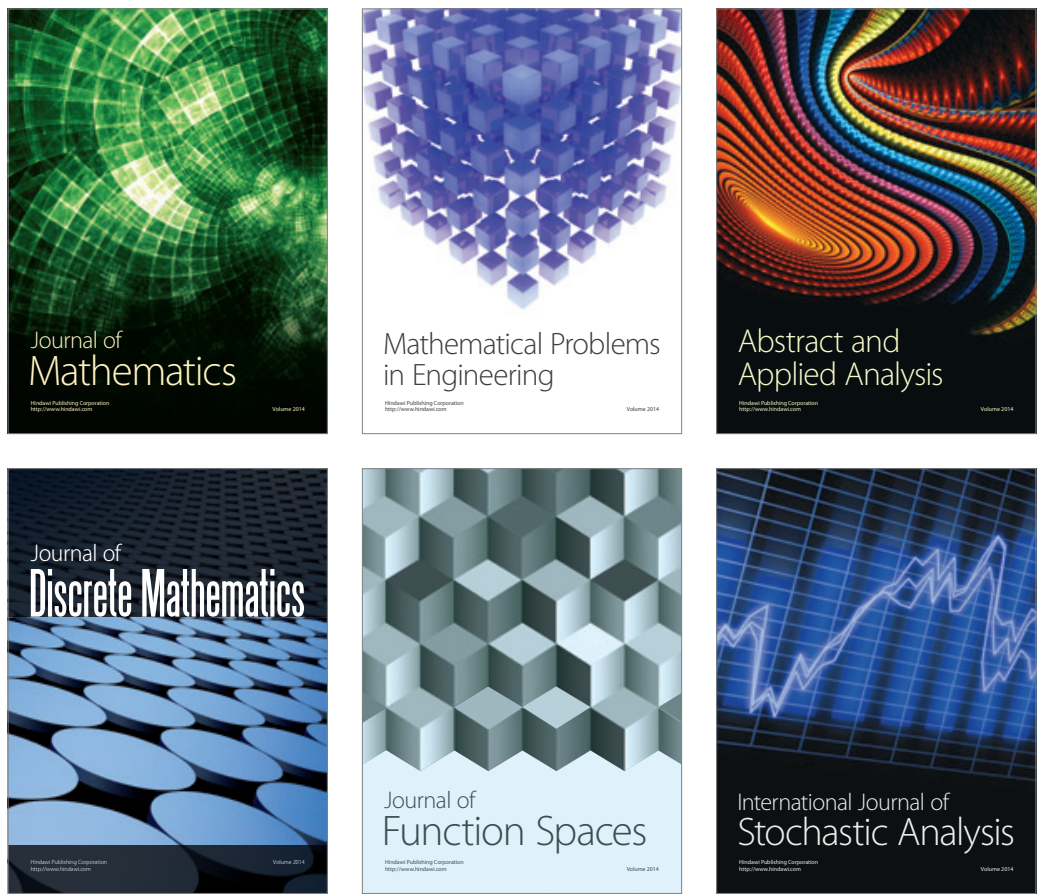

Journal of

Function Spaces

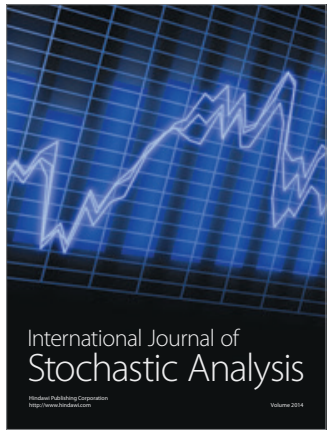

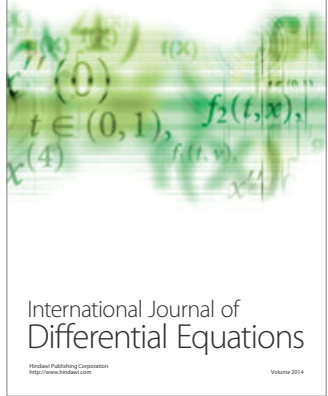
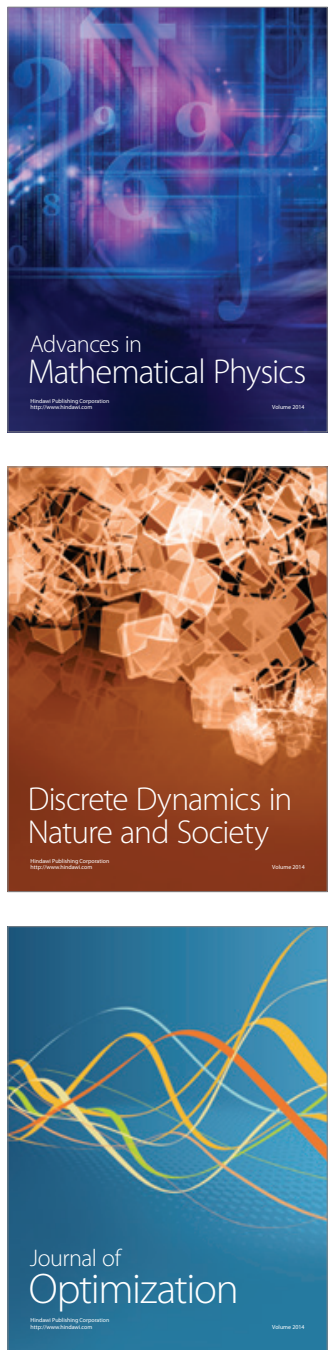